\title{
EFECTOS DE LA TRANSICIÓN DE UN SISTEMA DE EDUCACIÓN INTERCULTURAL BILINGÜE A UN SISTEMA DE EDUCACIÓN MONOLINGÜE, EN ESTUDIANTES DE $5^{\circ} \mathrm{GRADO}^{12}$
}

\author{
Edgar Salazar Francis ${ }^{13}$ \\ Marbel Baltodano Baltodano ${ }^{14}$
}

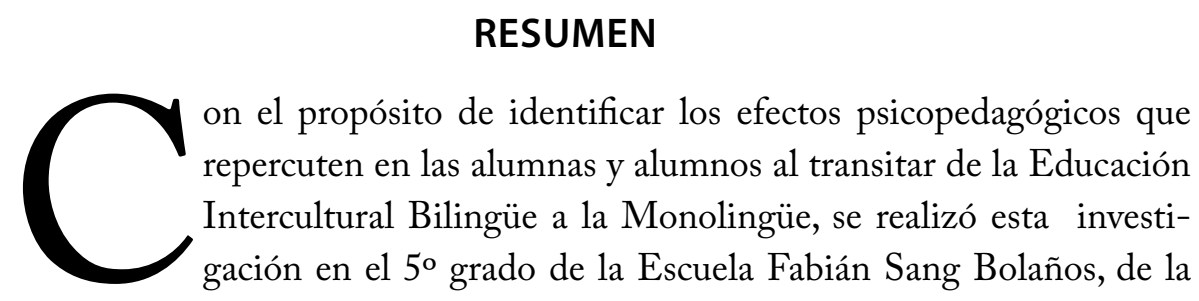
comunidad de Lamlaya en el municipio de Puerto Cabezas, Región Autónoma Atlántico Norte, RAAN, durante el primer semestre del año escolar 2005.

Este estudio es de tipo cualitativo, descriptivo, de carácter propositivo y de eje transversal, en él se tomó en cuenta información referente a aspectos pedagógicos, didácticos, estrategias metodológicas, técnicas de enseñanza - aprendizaje y de evaluación, Para lograr los objetivos planteados se realizaron entrevistas a docentes, alumnos y alumnas, además, se hicieron observaciones de clase directamente a pie de aula con el fin de recopilar datos cualitativos y cuantitativos apegados lo más cercano a la realidad del proceso enseñanza - aprendizaje.

Entre los resultados obtenidos se destaca que el Ministerio de Educación, no cuenta con un diseño curricular basado en principios científicamente establecidos de transición, entre la Educación Bilingüe a la Monolingüe, no se aplican supervisiones y seguimientos periódicos en las aulas de clase, planificación de clase fuera del contexto y entorno sociocultural del educando, escasez de textos y materiales didácticos para el desarrollo de la clase y la/el docente no está capacitada(o) para realizar actividades que permitan empalmar la educación Intercultural Bilingüe con la Monolingüe.

12 Investigación realizada por URACCAN Recinto Bilwi.

13 Lic. Educación Intercultural Bilingüe., URACCAN, Bilwi - Puerto Cabezas - RAAN., esalazarf06@ yahoo.es

14 MSc. Directora Académica URACCAN, bmarbel@gmail.com. Tutora de la investigación. 
Como efectos psicopedagógicos que afrontan las alumnas y alumnos podemos destacar los siguientes; dificultad en la asimilación de los contenidos en estudio, poco desarrollo en las habilidades para la lecto-escritura, dificultades en las operaciones básicas en matemática, altos niveles de abstencionismo a la hora de la participación en clase, poco desarrollo lingüístico en español como segunda lengua, alumnas y alumnos repetidores y memoristas, alto grado de ausentismo y deserción escolar, lo que, por ende, incide en el bajo rendimiento académico de las alumnas y alumnos.

Producto de esta investigación se brindan algunas sugerencias en los aspectos pedagógicos, metodológicos y didácticos, además de algunas propuestas concretas que contribuyan a superar estas debilidades y dificultades identificadas.

Los objetivos que guiaron el trabajo pretenden identificar y analizar los principales efectos psicopedagógicos que asume el educando al pasar de un sistema de enseñanza bilingüe a un sistema de enseñanza Monolingüe y proponer estrategias metodológicas que contribuyan a facilitar dicha transición.

\section{INTRODUCCIÓN}

La educación en Nicaragua, en los últimos años ha tenido grandes cambios desde el punto de vista organizacional, sus políticas, sus lineamientos; así también, la práctica docente en las aulas de clases, lo que se ha revolucionado desde la perspectiva y los retos del nuevo milenio que se han asumido.

En la Costa Caribe Región Norte, la población en su mayoría es Miskitu hablante, o sea que pertenecen al pueblo indígena Miskitu quienes tienen su propia forma de transmitir sus expresiones culturales partiendo de su cosmovisión, sus valores, normas de convivencias sociales y lingüísticas.

A consecuencia de los modelos de educación exógena que se han aplicado en las comunidades de las Costa Atlántica se revela que las alumnas y los alumnos identifican algunos elementos positivos tales como el hecho de que: "La escuela enseńa a leer, escribir y hablar un poco el español, así como otros conocimientos en matemática y algo sobre ciencias e historia de otros pueblos. Además, el haber estudiado en la escuela les sirve para comunicarse con los que hablan sólo español y otros idiomas.

Sin embargo, hay que considerar que, una de las finalidades fundamentales, si no la esencial de la educación, es desarrollar en el individuo procesos para construir conocimientos, desarrollar habilidades, aptitudes, actitudes, valores, capacidades y destrezas 
para enfrentar y dar respuesta a la vida, y todo proceso de formación debe realizarse de forma ordenada, de manera secuencial y concatenada, respetando la jerarquía de contenidos, acorde al nivel de la niña y el niño, para lo que la planificación desempeña un papel importante en la evolución del proceso enseñanza - aprendizaje.

Ujukam G (1997) indica que en estudios realizados en otros contextos (México, Canadá, Perú), han demostrado que estudiantes que transitan de un sistema de enseñanza bilingüe intercultural a un sistema de educación monolingüe, han demostrado un bajo nivel de aprovechamiento académico y aumento de la deserción y el ausentismo escolar, ya que la escuela no contribuye a la formación de las/los niños(as) en la cultura indígena, ni a fortalecer las instituciones comunitarias, los programas y los libros de textos cuentan con grandes vacíos ya que no enseñan nada de las culturas autóctonas, no explican como vivieron nuestros antepasado y qué nos han dejado para la vida actual y el futuro.

El modelo de Educación Intercultural Bilingüe (EIB), en su aplicación en las escuelas de la Costa Caribe Norte, ha tenido limitaciones para poderse implementar en toda la educación primaria y únicamente llega hasta el cuarto grado. Por esta razón las/los alumnas(os) tienen que continuar sus estudios en la modalidad monolingüe, y esta situación se viene presentando desde que se implementó la EIB desde 1984.

La adaptación a un nuevo sistema de enseñanza perjudica gravemente el aprovechamiento académico de las/los estudiantes, así también en el nivel de $5^{\circ}$ grado, el ausentismo, la deserción escolar y el rendimiento académico durante el primer semestre de cada año es alarmante, en comparación con los otros períodos de evaluación.

En educación, la transición de un sistema Bilingüe a uno Monolingüe se debe realizar con mayores y mejores niveles de planificación basados en los principios científicos, para no crear secuelas traumáticas ni psicológicas en las/los estudiantes, especialmente si son de niveles bajos según la edad, por lo que se realizó esta investigación en la escuela Fabián Sang Bolaños de la comunidad de Lamlaya.

A través de esta investigación se logró identificar algunos efectos de índole psicopedagógico y de planificación educativa que sufren las/los alumnas(os) al transitar de una Educación Bilingüe a una Monolingüe. Ante estos hallazgos se propone algunas recomendaciones y estrategias metodológicas a los docentes, que contribuyan a superar el rendimiento académico y obtener mayor y mejor aprovechamiento escolar por las/los estudiantes que se encuentran ante esta situación. 


\section{REVISIÓN DE LITERATURA}

Venezia (2003) afirma que los estudiantes se han visto relegados en un segundo plano a la hora de la participación, y esta consiste en la mayoría de los casos en repetir, ya que la mayor parte del tiempo es la/el maestro(a) el que habla y participa en la clase.

German (1997)) indica que la repetición en la lengua del educando crea retraso en el aprendizaje de la segunda lengua $\mathrm{Al}$ respecto, Freeland (1994) expresa que esta es una estrategia que tiene que ser contraproducente durante el desarrollo de la clase, ya que metodológicamente no es recomendado repetir inmediatamente la lección en la lengua materna de las/los alumnas(os), porque estos no le brindarán importancia al aprendizaje de la segunda lengua, y agrega que lo anterior se agrava en el caso de la alumna(o) del Programa de Educación Bilingüe Intercultural, PEBI, en la que los docentes encuentran grandes dificultades para avanzar en los contenidos programáticos argumentando que los alumnas y alumnos no asimilan los contenidos desarrollados por el poco dominio del espańol, y para cubrir el programa monolingüe espańol los docentes avanzan sin respetar el ritmo de aprendizaje de cada uno de los educandos.

Venezia (2003) afirma que en primer lugar se deberán crear espacios para la libre expresión de los educandos sobre temas de su interés y partir de sus conocimientos previos sobre el tema en estudio, para crear la motivación necesaria y así lograr un mayor aprovechamiento académico.

Después que los estudiantes aprenden los mandatos orales, aprenden a leerlos y escribirlos. Más adelante, cuando los estudiantes empiezan a hablar y leer con más perfección, las actividades incluyen juegos y resolución de ejercicios y problemas, según su nivel, al respecto Ujukam (1997) expresa que los docentes combinan elementos, dan las orientaciones para que los estudiantes desarrollen flexibilidad y comprensión ante la asimilación de los nuevos conocimientos. Además, según Freeland (1994) las medidas de evaluación no se deben medir cuantitativamente, sino cualitativamente, o en términos de la gama de funciones comunicativas que dominan. Dado que esto no se hace, el empalme sigue presentando dificultades para los educandos de cualquier grupo lingüístico ante esta situación.

Montes (1999) dice que la educación debe contar con la introducción de los saberes ancestrales en los planes y programas de clase o aula escolar y Acunzo Mario (1991) manifiesta al respecto, la necesidad de que los planes y programas puedan adecuarse a las necesidades educativas de la comunidad, a su vez, Cunningham (1999) expresa que la educación debe mantener y cultivar las tradiciones, cultura y formas de educación endógena, con la intención de partir de los saberes previos o ancestrales, el entorno de la comunidad y extenderse al mundo, además deberá organizar las tareas cotidianas y los ambientes para estimular y facilitar la comunicación, así como la práctica pedagógica en 
las que multiplicará las situaciones comunicativas, recurriendo con mayor frecuencia a las actividades grupales.

Freeland (1994) expresa que los educandos enfrentan diversos grados de frustración y desmoralización que eventualmente conducen al fracaso escolar. En relación a esto el docente no está capacitado metodológicamente para realizar el empalme entre el sistema de educación Bilingüe al Monolingüe y se continúan aplicando estrategias metodológicas tradicionales en el aula de clase.

En algunos casos, los estudiantes no cuentan con poyo de sus familiares, afectados por las condiciones económicas, además los bajos niveles escolares de los familiares no les permite apoyar en sus estudio a sus hijas e hijos, también han sido discriminados por su idioma, factores que los obligan a retirarse de la escuela, aumentando el índice de deserción escolar y la repitencia, Venezia (2003).

Traña (1998) expresa que la evaluación comprende un balance y una apreciación crítica y valorativa de toda la operación de aprendizaje, con miras a efectuar rectificaciones y mejorar el proceso de enseñanza, a través de la inserción en la planificación de todos aquellos elementos identificados durantes los procesos evaluativos ya sean iniciales o durante el proceso. Además, Carreño (1998), menciona que la evaluación es el conjunto de operaciones que tiene por objeto determinar y valorar los logros alcanzados por las/los alumnas/os en el proceso de enseńanza - aprendizaje, con respecto a los objetivos planteados en los programas de estudio, entonces se deberá tomar en cuenta el aprendizaje individualizado de cada uno de los estudiantes en este proceso, a través de la planificación y evaluación de manera sistemática los nuevos conocimientos.

La finalidad de la evaluación diagnóstica, es recabar información inicial sobre los conocimientos que nuestras/os alumnas(os) poseen en torno a los estudios del curso, qué información, conocimientos, habilidades y destrezas tiene al iniciar una unidad de aprendizaje, para diagnosticar qué problemas pueden ser la causa de la relación eficienciadeficiencia en el comportamiento del educando, Sola (1989).

Por su parte, Carreño (1998) define la evaluación como el conjunto de operaciones que tienen por objeto determinar y valorar los logros alcanzados por las alumnas y alumnos en el proceso de enseńanza - aprendizaje, con respecto a los objetivos planteados en los programas de estudio. Sola (1989) indicó que la evaluación es un proceso sistemático, no dependiente de un maestro sino de la eficiencia en que se logran los objetivos educacionales propuestos para una lección, curso o grado.

El plan de clase es el documento que facilita al docente organizar y dirigir el desarrollo del proceso enseñanza - aprendizaje. Es el producto de la preparación y reflexión sobre la forma más efectiva de desarrollar la clase, además, hay que destacar que la importancia de un plan de clase se debe a la forma cómo se dirige y organiza las actividades de la clase, en base a objetivos pedagógicos en estrecha relación con el contenido de estudio. 
En este sentido, Mialaret (1999) expresa que la planificación educativa debe incluir la transmisión de la cultura del individuo, que se enseñen los valores, principios culturales, tradiciones, idioma, cosmovisión; además, la práctica ha demostrado que los educandos presentan mayor interés cuando la educación parte de sus experiencias y conocimientos previos, los que deberán ser tomados en cuenta a la hora de desarrollar la clase, no importa en cual idioma sea. Ujukam (1997) coincide en este planteamiento al expresar que dan muy buenos resultados con niñas y niños que continúan sus estudios en una segunda lengua; partir de la descripción de una fotografía, lámina de escenas típicas de su comunidad, observar fotos, láminas de actividades que se realizan en su comunidad y se les pide que lo describan, con lo que se estará partiendo de los conocimientos pertinentes de cada uno de los estudiantes.

Sobre la metodología, Ujukam (1997) afirma que ésta señala el encadenamiento lógico de ejercicios graduados para obtener un fin; los métodos, las técnicas y los procedimientos de enseñanza que se utilicen serán activos, funcionales, accesibles a los intereses y las capacidades de los educandos.

Freeland (1994) indicó al respecto que el sistema de educación en la región de la Costa Caribe, en su totalidad parece adolecer de una cierta fragmentación y falta de secuencia entre sus distintos niveles y etapas de aprendizajes, la mayoría de los maestros y maestras parecían tener poco o ninguna noción de cómo se integraba su propia labor docente dentro de la lógica de su grado.

\section{MATERIALES Y MÉTODOS}

Este es un estudio de caso cualitativo en que se aplicaron entrevistas con preguntas abiertas, observaciones y su función final es la de explicar e interpretar las situaciones estudiadas, además, estudia grupos en los que es posibles realizar observaciones directas e indirectas, descriptivo de carácter propositivo y de eje transversal; la población de estudio lo comprendieron 24 estudiantes del $5^{\circ}$ grado de la sección "A", al director y docente de la escuela Fabián Sang Bolaños, de la comunidad de Lamlaya.

\section{VARIABLES EN ESTUDIO}

- Efectos Psicopedagógicos

* Lingüístico

* Aprovechamiento académico

* Deserción y ausentismo escolar

- Metodología de enseñanza - aprendizaje 
* Planificación didáctica y técnicas de evaluación

* Estrategias, métodos y técnicas de enseñanza

* Empleo de material didáctico

\section{INSTRUMENTOS QUE SE UTILIZARON PARA LA OBTENCIÓN DE LA INFORMACIÓN}
a. Guía de entrevista al maestro(a) (Ver anexo. Página 53)
b. Guía de entrevista al director (Ver anexo. Página 53)
c. Guía de entrevista al estudiante (Ver anexo. Página 54)
d. Guía de observación del ambiente escolar (Ver anexo. Página 55)

Los que se seleccionaron considerando que son los más apropiados para este tipo de investigación dado su carácter cualitativo. Las estrategias de aplicación de los instrumentos para la obtención de la información, se basó en entrevistar al director del centro quien accedió muy amablemente a brindar la información. Así también, la entrevista del docente del $5^{\circ}$ grado, se efectuó fuera de las horas laborales, esto con el propósito de no afectar el desarrollo normal de las clases. El docente colaboró muy amablemente, durante la entrevista se pudo notar que existe un alto espíritu de superación en su forma de expresar lo que se vive en el aula de clase, pero que casi nunca ha tenido la oportunidad de una capacitación que le ayude a superarse profesionalmente.

Las entrevistas llevadas a cabo con las/os alumnas(os) se efectuaron durante diversas sesiones y jornadas de trabajo a lo largo del período investigativo, procurando en la medida de lo posible no interrumpir ni afectar las sesiones de clase, un aspecto muy importante a destacar en las entrevistas es que la gran mayoría de las/os alumnas(os) entrevistadas(os) respondieron las preguntas en español con mucha incoherencia, entrelazadas con palabras en miskitu, por lo que se tuvo que replantear las preguntas en miskitu para obtener mayor información que contribuyera con la investigación.

Para el proceso de observación del ambiente escolar a pie de aula en el $5^{\circ}$ grado, se planificaron y desarrollaron tres visitas a la semana, en diversas horas de la jornada escolar, toda la información obtenida fue registrada en la libreta de campo. En principio se conversó con el director y docente del centro y grado en mención, la observación realizada no fue participativa de parte del investigador, ya que este consistió en observar y anotar las vivencias de la clase como se manifestaron, las jornadas de observación se realizaron con el propósito de obtener la mayor información posible de todas las áreas y asignaturas del currículum en estudio. Durante este proceso, al inicio se observó mucha inhibición en la participación tanto de las/os alumnas(os) y del maestro; pero, transcurrido el tiempo se acostumbraron a la presencia del investigador y esta primera impresión cambio en al aula 
de clase y se pudo obtener datos cualitativos y de trascendencia para esta investigación, se observaron clases en el aula y al aire libre.

\section{Procesamiento de datos}

Para el procesamiento de los datos se aplicó la técnica del palote, cuadros de comparación siguiendo el orden de las entrevistas realizadas y las observaciones de clase efectuadas a pie de aula y contrastados con el marco referencial según las variables y los objetivos planteados.

\section{RESULTADOS Y DISCUSIÓN}

\section{EFECTOS PSICOPEDAGÓGICO}

\section{Lingüístico}

Con relación a la participación durante la clase, se observó que debido al poco dominio del español, la intervención de los educandos es limitada, ya que se centran únicamente en realizar lectura y escritura de manera mecánica, y cuando leen en español no analizan lo que leen, se vuelven memoristas y repetidores de lo que dice el profesor. Además los educandos demuestran temor en participar o hablar porque tienen poco dominio del español, y ellos creen que se expresarán mal, ya que cuando uno les habla en español como que tienen pena y responden en Miskitu cuya lengua tienen mayor dominio y seguridad de no equivocarse en las respuestas.

En relación al idioma en que desarrolla la clase se observó que casi siempre las clases son en español porque así están diseñados los programas de quinto grado, pero inmediatamente el/la docente repite la lección en la lengua de los educandos (Miskitu), fenómeno que crea dependencia a la lengua materna y estos se desobligan a la hora en que el profesor explica en español.

Sobre el aprendizaje de sus educandos la docente expresa: "La mayoría de estudiantes tienen grandes dificultades en la asimilación de los contenidos en especial en español y matemática ya que ellos no tiene su propio texto, la mayoría hablan sólo en miskitu y eso es un problema bien serio ya que cuando se realizan preguntas en español no entienden y no pueden responder, y si responden es en un 'mal español' en la que confunden género, número y sintaxis, y la mayoría de los casos las respuestas son incorrectas, pero si les preguntan en su lengua materna ellos responden al instante". Manifestación que se observa debido a que en el Programa de Educación Bilingüe Intercultural (PEBI), al parecer no lograron desarrollar las habilidades básicas en los niveles anteriores según normas y orientaciones de una Educación Intercultural Bilingüe. 


\section{Aprovechamiento Académico}

Sobre la participación de las/los alumnas(os), en las diferentes asignaturas esta se concentra en resolver ejercicios en la pizarra, leer algún texto en español, todo esto con contenidos fuera del contexto del educando, escribir la lección de la pizarra en su cuaderno o transcribir la lección del texto al cuaderno, acción que les consume tiempo y no se aprovecha para analizar e interpretar la lectura, comentar los ejercicios resueltos y los textos leídos, ya sea en equipo o con la ayuda del maestra(o).

Referente a qué les gusta de las clases que reciben cada día, ellos han manifiestado que les gusta la clase de matemática porque en ella se aprende a sumar y restar, les gusta la clase de español porque en ella aprenden las sílabas, lectura y escrituras y además quieren aprender a hablar y escribir bien en español, les gusta que los pongan a escribir en su cuaderno, les gusta cuando el profesor explica la clase en miskitu y ellos entienden el tema, algunos manifiestan que le gusta cuando pasan a la pizarra a resolver algún tipo de ejercicio cuando ellos entienden, pero en estos casos el maestro no aplica las combinaciones básicas de los elementos de aprendizaje que consiste en la teoría y el descubrimiento de nuevos conocimientos a través de la práctica, para crear un mayor nivel de desarrollo cognoscitivo de las oraciones y contenidos en estudio.

Dada las características de las alumnas y alumnos en esas edades, se deben mantener en constante actividad pero ésta debe ser planificada de manera ordenada y secuencial según objetivos y metas trazadas, previendo el tiempo del que se dispone, ya que se observó que en la mayoría de las actividades que se realizan en el aula de clase, no existe alguna planificación ni evaluación, casi todo se realizaba de manera improvisada, lo que crea inseguridad a la hora del aprendizaje.

Referente a cómo considera que es el rendimiento académico de los alumnos y alumnas durante este primer semestre, el profesor manifestó: "El rendimiento es regular ya que está entre los 60 y el $65 \%$ de aprobado en general, lo que es muy bajo; pero esto es debido a que cuando se les asigna tareas en casa las alumnas y alumnos no las cumplen y así pierden puntos", pero en este fenómeno incide la falta de comprensión de los contenidos en estudio.

También durante las evaluaciones la mayoría reflejan notas reprobadas o si no con nota baja, y se observó que las evaluaciones se centran más en aspectos teóricos, memorísticos y referenciales a los textos que utiliza en el aula de clase, y en ellas no se refleja la evaluación en cuanto al dominio de las habilidades lingüísticas, y con temas relacionados a su entorno sociocultural.

En cuanto al nivel de adecuación del contenido al entorno sociocultural del estudiante, se constató que algunos docentes no realizan ningún tipo de adaptación o adecuación de los contenidos programáticos a la realidad del educando y la comunidad, ni considera la cosmovisión de estos, en la mayoría de los casos, habló de volcanes, lagos, ríos, núme- 
ros y fórmulas, ciudades y personas, animales, maquinarias, narró algunos cuentos; pero, casi nunca lo relacionó con la realidad de la nińa y el niño del salón de clase, estos fueron contenidos extraídos directamente del texto, y además, la mayoría de los contenidos estudiados quedaron en el nivel de conocimiento, y se descuidó en muchas ocasiones el desarrollo de habilidades, no pasó del nivel de saber al de saber hacer y saber crear.

Se constató que, el maestro no cuenta con la capacidad pedagógica necesaria para poder realizar algún tipo de adecuación y adaptación de los contenidos al entorno de las nińas y niños, con el propósito de disminuir los efectos negativos que sufren estos durante esta importante transición.

Con relación al rendimiento académico, los educandos manifiestan que es muy bueno, aunque llevan dos o tres asignaturas reprobadas. Existe el fenómeno que la mayoría desconoce su rendimiento académico al corte semestral, ya que no han recibido sus evaluaciones y parecen desconocer el sistema de calificaciones que emplea el docente para evaluarlos o es que no se les ha dado a conocer, porque una minoría argumenta que ellas/ os participan en clase y creen que su rendimiento es bueno o sea aprobado. Otro fenómeno que ocurre, es que durante las evaluaciones el docente les da a conocer la acumulación de nota cuantitativa antes del examen, diciéndoles cuanto les falta para llegar a obtener los 60 puntos que es la nota mínima para aprobar, lo que considero que no es correcto ya que predispone psicológicamente al estudiante para llegar a lograr esa nota y no esforzarse por obtener mayores calificaciones.

\section{Deserción y Ausentismo Escolar}

Referente a las deserciones y las causas que las provocaron, se logró constatar que en este nivel iniciaron estudiando 24 alumnos y alumnas de entre 14 y 17 años, de los cuales al concluir la investigación de campo, al corte del primer semestre del año lectivo se contabilizaron nueve (9) deserciones lo que equivale al $38 \%$, y entre las principales causas se registran que la mayoría se deben por problemas lingüísticos o sea poco o ningún dominio del español como segunda lengua, habilidades en la lecto-escritura al parecer no desarrolladas durante los niveles anteriores en el PEBI, según se pudo observar, se enseña únicamente en Miskitu, lo que incide negativamente en cuanto a las exigencias pedagógicas del $5^{\circ}$ grado monolingüe, esto redunda en que casi todos los retirados llevaban más de tres asignaturas reprobadas con notas de 20 y 40 puntos al corte semestral, lo que los desmotivó a continuar con sus estudios.

Otro problema visualizado es el económico, ya que cuatro retiradas/os eran de las comunidades del litoral sur, quienes enfrentaban serias dificultades de alimentación, vestuario y demás necesidades personales básicas que fueron manifiestas por los estudiantes afectados; además, durante las visitas se observó que los padres de familia no asisten a las reuniones y actividades que realiza la escuela. 
Se determinó la existencia de un alto nivel de ausentismo, llegadas tarde e inasistencia de parte del alumnado, manifestaciones debidas a diversos factores y asociados con la práctica pedagógica, ya que el empalme realizado entre la educación bilingüe al monolingüe no es el más adecuado, por consiguiente la escuela no llena las expectativas de aprendizaje del alumnado, lo que ocasiona un alto grado de desmotivación y frustración en la niñez.

Además, se verificó que en la mayoría de los casos no se realiza ningún tipo de visita domiciliar para identificar cuales pueden ser las causas de las deserciones, ausentismo y tardanza de sus alumnas y alumnos en el seno de la familia, para facilitar la mediación de manera oportuna y brindar algunas orientaciones según sea el caso.

\section{METODOLOGÍA DE ENSEÑANZA - APRENDIZAJE}

\section{Planificación didáctica Y TÉCnicas de eValuación de la ClaSe}

Se aplicó la prueba diagnóstica inicial, para determinar el nivel de entrada de los alumnos y alumnas y así realizar una graduación y adecuación de los contenidos, ésta se aplicó al inicio del año escolar en casi todas las asignaturas, y los resultados que se obtuvieron fueron que la mayoría de los estudiantes presentaron dificultad en el área de espańol, ya que no saben leer fluidamente, no analizan lo que leen, presentan poco dominio del español, dificultades en la caligrafía y ortografía, lentitud en la escritura.

En el área de matemática, no demuestran habilidades para resolver ejercicios aplicando las cuatro operaciones básicas. En el área de educación física, presentaron dificultades a la hora de realizar los ejercicios de resistencia y velocidad. Todos estos resultados obtenidos no fueron tomados en cuenta al momento de la planificación de parte del docente ya que se constató que los contenidos desarrollados no tuvieron alguna adecuación según los resultados obtenidos de la prueba diagnóstica.

Los resultados del rendimiento académico durante los primeros cortes evaluativos fueron muy por debajo de los niveles deseados, con un alto grado de reprobados especialmente en el área de español y matemáticas.

El paso del sistema bilingüe al sistema nacional monolingüe, se determinó que no ha sido objeto de planificación alguna en ninguna de las fases y etapas del programa, referente a esto se constató que, el Ministerio de Educación, no tiene un plan donde se manifieste que desde quinto grado qué deben de seguir, desde el inicio del año escolar, en relación a un programa de transición o directrices en los aspectos pedagógicos y didácticos, desde el ámbito Bilingüe al Monolingüe, que tenga el propósito de mitigar algunos de los efectos psicopedagógicos que afectan a los educandos ante esta situación, que se agrava con incidencias negativas, en las escuelas rurales, debido a que las y los docentes, en su mayoría empíricos, no están capacitados para enfrentar este empalme o transición. 
Además, se verificó que el docente, cuenta con orientaciones claras y precisas en cuanto a no realizar pérdida de horas clases en repaso de contenidos, sino más bien avanzar en el desarrollo programático, lo que a mi juicio tiene grandes desventajas para el alumnado ya que ellas/os no logran consolidar el nuevo conocimiento debido al poco dominio del español y se crean grandes lagunas de conocimientos que de por sí ya traen desde los grados inferiores al $5^{\circ}$ grado.

$\mathrm{Al}$ respecto se constató que la función de la planificación no logra los objetivos trazados ya que los docentes planifican de acuerdo al programa de quinto grado que es su guía, además el director revisa los planes de clase cada viernes y no diariamente, pero si encuentra algún error hace las observaciones pertinentes; pero, esto ocurre cuando ya se ha desarrollado la clase durante la semana.

Sobre la importancia y funcionabilidad de la planificación docente se constató que la/el maestra(o) planifica su clase diariamente según formato y normativa brindada por el Ministerio de Educación, pero esta planificación casi siempre no se logra desarrollar durante el período de la clase, ya que las actividades planificadas no se ajustan al tiempo destinado para la clase según horario escolar, (45 y 90 minutos según la asignatura), y estas quedan inconclusas y por lo tanto los aprendizajes quedan truncados o a medio camino, porque en la siguiente clase se inicia otro contenido e igualmente queda inconclusa, repitiéndose así el ciclo que afecta el rendimiento académico, más cuando la clase es en español, en el que presentan mayores dificultades de aprendizaje, aunque se les asignen los contenidos como tarea en casa. Además, el docente manifiesta que invierte gran cantidad de tiempo planificando sus clases y los resultados no son los deseados, ya que siempre hay un gran número de educandos reprobados.

En relación a la integración de los elementos culturales durante la planificación de la clase, se constató que no se toma en cuenta los elementos culturales, lingüísticos y la cosmovisión de los educandos en relación al entorno inmediato de la comunidad, lo que se manifiesta en desinterés, desmotivación, y esto puede estar incidiendo en los altos índice de deserción y ausentismo escolar.

Se constató que el docente realiza evaluaciones diagnósticas al inicio del año escolar, $y$ en determinados contenidos de algunas asignaturas durante el proceso educativo; pero, también se verificó que en la mayoría de los casos esta evaluación descubre grandes debilidades y deficiencias de aprendizaje, y para superar esta realidad el docente no toma en cuenta los resultados durante el proceso de planificación y desarrollo docente de la clase.

Además, aplica evaluaciones sistemáticas en las que asigna algún puntaje cuantitativo al cumplimiento de tareas, a la participación en clase y a las actividades extra curriculares que realizan, cuando resuelven ejercicios en la pizarra, así también realiza preguntas orales en algunas ocasiones. 
Hay que destacar que en la mayoría de las clases observadas el docente no aplicó ningún tipo de evaluación, y no existe un registro de las participaciones de los educandos en la clase, para poder cuantificar el puntaje acumulado por cada uno de ellos.

Se verificó que aplica la evaluación sumativa o al finalizar un determinado período mensual, esta es meramente cuantitativa. Sin embargo, se percibió que no existe una planificación sistemática del proceso evaluativo ya que en muchas ocasiones improvisó actividades evaluativas.

\section{Estrategias, Métodos y Técnicas de Enseñanza.}

Con relación a las estrategias y técnicas de enseñanza, los resultados de la investigación determinan, que la mayoría de las clases observadas fueron expositivas, haciendo uso excesivo de la pizarra para resolver ejercicios, especialmente durante la clase de matemática, además emplea mucho el dictado de lecciones, la trascripción de textos completos al cuaderno, emplea la lectura oral de parte de los educandos, pero estas lecturas no son comentadas ni analizadas en el seno del grupo de clase.

En la mayoría de las clases observadas, el docente no orientó el objetivo de la clase, o sea que no había ningún fin para el estudio de los contenidos; así también, no señaló ni dijo qué estrategia metodológica y técnicas utilizaría para el desarrollo de la clase; la mayoría de las veces se observó la improvisación de actividades que quedaron inconclusas, de repente ponía a saltar a las alumnos y alumnos y los movía de un lugar a otro sin ningún fin ni relación con los contenidos que se estaban desarrollando y esto, muchas veces desorientó a los participantes en la clase.

En algunas ocasiones, utiliza técnicas grupales, pero debido a la falta de orientación de los objetivos, las actividades a realizar y los pasos a seguir en la técnica, los alumnos y alumnas desaprovecharon el tiempo, la actividad quedó inconclusa y el maestro no realizó las conclusiones ni la evaluación pertinente de la clase, con lo que no se logra visualizar la consolidación del nuevo aprendizaje cognoscitivo, ni permite identificar los posibles errores. Se considera que toda clase debe tener un fin y un objetivo pedagógico que debe darse a conocer a los alumnos y alumnas con anticipación, así también los métodos, técnicas y estrategias que se utilizarán para alcanzar a desarrollar los conocimientos, habilidades, destrezas y valores en los alumnos y alumnas.

En relación a los conocimientos que posee el docente sobre los contenidos de cuarto y sexto grado y cómo estos se interrelacionan con su actividad docente en el aula de quinto grado, éste manifestó que en algunos casos, como en la matemática, se relacionan cuando se estudia la propiedad asociativa, en la asignatura de español y ciencias naturales siempre debe existir alguna relación; pero, para el desarrollo de sus clases manifiesta que no existe ninguna relación ya que no los toma en cuenta y no realiza ningún tipo de ade- 
cuación o graduación de los contenidos, ya que para eso él tiene un programa que es la guía para planificar su clase.

Por otro lado, durante el semestre en ninguna ocasión tuvo la oportunidad de reunirse con los docentes que atienden a los alumnos del cuarto y sexto grado en el mismo colegio, para poder identificar los contenidos comunes a estos grados y así poder realizar una graduación y adecuación de los contenidos para que sean más accesible a los alumnos y alumnas, y prever una menor incidencia en los efectos psicopedagógicos que afectan a los educandos en estos dos niveles.

Se considera que debería existir un espacio y un tiempo asignado para que los maestros y maestras puedan realizar encuentros en los que se propicien intercambios de experiencias y conocimientos, para analizar los diferentes programas de cada grado y así tener una visión más generalizada de los contenidos en cada grado y poder realizar una graduación, adecuación y contextualización de los mismos en cada grado a la hora de la planificación de la clase, con el propósito de disminuir algunos efectos psicopedagógicos que puedan afectar a los alumnos y alumnas.

Con relación a las capacitaciones y actualizaciones pedagógicas que recibe el maestro(a), se constató que únicamente han recibido una, relacionada al uso y manejo de los textos escolares, pero que en casi nada le ha servido ya que no cuenta con los textos escolares, lo que limita el desarrollo de la clase. Además no se ha recibido ninguna visita ni capacitación de parte de los técnicos del Ministerio de Educación. El único que realiza las visitas de supervisión es el responsable del centro, una vez al mes, en la cual hace el llenado de un formato, pero esta supervisión no deja resultados positivos, ya que nunca ofrece alguna sugerencia o recomendación para superar las dificultades pedagógicas. Se constató que en muchas ocasiones el docente realiza un plus esfuerzo para brindar una enseñanza adecuada y pertinente, pero casi siempre se encuentra con obstáculos socioculturales y económicos que le impiden cumplir con éxito su labor docente.

\section{Empleo de Materiales Didácticos}

El material de apoyo o didáctico debe propiciar situaciones significativas que permitan al alumno y la alumna participar espontáneamente en una conversación, discusión o "lluvia de ideas" sobre el tema o asunto que va a tratar, puede ser un cuento, una anécdota, una cita bibliográfica, un cuestionario, una guía de lectura, una ficha de contenido. En este sentido, se observó que los materiales utilizados por el docente fueron algunos textos de cuarto y quinto grado, algunas láminas y papelógrafos con información elaborada por él, pero en la mayoría de las clases observadas no utilizó ningún tipo de material didáctico además de la pizarra. 
En la clase de matemáticas por lo general las/los alumnas(os) utilizan su cuaderno y la pizarra para resolver ejercicios, esto ocurre ya que la escuela no cuenta con los materiales y medios de enseñanza que harían la clase más entretenida y comprensiva. Además, el centro no cuenta con biblioteca en la que puedan ir ha realizar consultas o algún tipo de investigaciones sencillas que se les pueda asignar, y si esto ocurre, tienen que ir hasta la ciudad de Puerto Cabezas para poder consultar en las bibliotecas que existen allí.

Sin embargo, las alumnas y los alumnos manifestaron que el único material que elabora el docente es el papelógrafo y con su uso entienden mejor el tema y participan más, les gusta porque parece que le ayuda al profesor a explicar mejor la clase aunque sea en español, algunos les gusta los contenidos, porque a través de ellos aprenden a dibujar, leer y escribir mejor en español.

\section{Características del docente}

El docente observado y entrevistado para efecto de esta investigación, es de etnia miskita, cuya lengua materna es el Miskitu, egresado de la escuela normal, con el título de maestro de educación primaria, con dos años de experiencias en educación, un año en la subdirección de la escuela y este año atendiendo el $5^{\circ}$ grado monolingüe, a la fecha no ha recibido ninguna capacitación o actualización pedagógica de parte del Ministerio de Educación. Durante las entrevistas y observaciones realizadas este se mostró muy abierto a colaborar para las observaciones planificadas.

Contrastando las características del maestro de EIB en la educación primaria, con el de ésta investigación se observan muy pocas similitudes, en principio él no tiene una formación académica para enfrentar situaciones pedagógicas y de Interculturalidad en el aula de clase, su formación profesional no le permite realizar adecuaciones en el proceso de enseñanza aprendizaje en $5^{\circ}$ grado monolingüe español.

\section{CONCLUSIONES}

La metodología empleada por la/el docente no permite crear espacio para la reflexión, creatividad y la auto gestión para formular otras vías de solucionar los problemas planteados y con esto formar estudiantes independientes y creativos, que enfrenten los nuevos retos de la vida con mayor decisión.

Hay dificultades de asimilación y desarrollo de habilidades y destrezas en español como en matemáticas; falta de habilidades en realizar lecturas interpretativas, en la solución de ejercicios y problemas aplicando las operaciones básicas en matemática.

Las/los alumnos no desarrollan las habilidades, capacidades, aptitudes y actitudes necesarias, para poder enfrentar los nuevos retos académicos del grado. Esto como con- 
secuencia de la poca preparación que tienen el docente en el manejo del programa de transito de educación intercultural bilingüe hacia la monolingüe.

La/el docente no cuenta con la preparación pedagógica para poder realizar un empalme pedagógico entre el modelo bilingüe al monolingüe, y no aplica las estrategias metodológicas adecuadas para la enseńanza - aprendizaje del educando que proviene de un programa de educación bilingüe intercultural.

La falta de planificación, incide generalmente en la improvisación de actividades que no tienen ninguna relación con los objetivos de la clase lo que desconcierta a los estudiantes y cada quien realiza lo que quiere sin ningún fin previamente trazado.

El aprovechamiento académico por parte del alumnado durante este corte semestral, refleja, datos cuantitativos bajos ya que el rendimiento académico general se encuentra en un $65 \%$ de aprobado.

Los niveles de adecuación curricular no se realizan en este grado, ya que los planes y programas se aplican tal a como vienen del nivel central, esto se debe a que el docente no cuenta con los elementos pedagógicos idóneos para realizar la integración de los elementos del entorno sociocultural de la comunidad en que vive el estudiante, la cosmovisión, los saberes ancestrales, cuentos y leyendas, las necesidades de educación y de producción, historia y origen de la comunidad.

Los estudiantes desconocen el sistema de evaluación y calificación utilizado por el docente y éste no les da a conocer qué mecanismos evaluativos está empleando en cada clase para evaluar, además predispone psicológicamente al educando a la hora de las pruebas parciales diciéndoles que únicamente necesitan cierto puntaje para aprobar con 60 que es la nota mínima para aprobar, con lo que el estudiante no se esfuerza por obtener una mayor calificación.

Entre los principales efectos de la transición que afectan los estudiantes de $5^{\circ}$ grado monolingüe, se encuentran los siguientes:

- Poco o ningún dominio del español hablado y escrito por parte de las alumnas y alumnos.

- Altos niveles de ausentismo y deserción escolar, principalmente durante los primeros cortes evaluativos.

- Los educandos demuestran poca capacidad de adaptación a las estrategias metodológicas que utiliza el docente en $5^{\circ}$ grado.

- Existe desmotivación total en los estudiantes que provienen del PEBI que se desarrollan en comunidades miskitas.

- Problemas económicos, para suplir las necesidades materiales y personales de los educandos. 
- La escuela no llena las expectativas educacionales con que ingresa un estudiante en el $5^{\circ}$ grado monolingüe español.

- Casi siempre en los primeros cortes evaluativos las calificaciones son bajas lo que motiva a la alumna y alumno a retirarse más pronto de la escuela.

- La escuela no cuenta con una política de retención escolar ya que mientras menos alumnos tengan que atender mejor se sienten los maestros debido a su bajo ingreso económico (salario).

El docente a la hora de desarrollar la clase no da a conocer los objetivos, estrategias, técnicas y actividades que realizarán para alcanzar los objetivos planteados y así medir las habilidades y destrezas desarrolladas por los estudiantes.

El director y/o responsable del centro no realiza acciones de acompañamiento en la supervisión al aula de clase, además, los planes de clase no los revisa para poder realizar algún tipo de recomendaciones pedagógicas a los mismos.

La escuela no cuenta con biblioteca y demás medios y materiales didácticos necesarios para poder brindar una clase con la calidad requerida, por lo que el profesor no emplea ningún tipo de material a excepción de algún papelógrafo que elabora de vez en cuando, y textos de otros grados inferiores.

El profesor desconoce hasta en un $90 \%$ los contenidos programáticos y el sistema de planificación y evaluación que emplean los docentes de $4^{\circ}$ grado, en el que la educación es bilingüe, lo que incide de manera negativa en la adecuación de los contenidos durante los primeros meses del año escolar, además los resultados de la prueba diagnóstica inicial no son tomados en cuenta para realizar una mejor planificación de su clase.

El Ministerio de Educación no cuenta con un diseño o planificación para brindar una atención psicopedagógica a los estudiantes que transitan del sistema de educación Bilingüe ( $1^{\circ} \mathrm{al} 4^{\circ}$ grado, Miskitu - español) al Monolingüe ( $5^{\circ}$ y $6^{\circ}$ grado español), para poder disminuir los efectos que perjudican a estos educandos durante esta transición.

Debido al empleo excesivo de la técnica expositiva, lectura oral, dictado y trascripción de textos en su cuaderno, los estudiantes ya predisponen lo que ocurrirá durante la clase, y esto trae como consecuencia el aburrimiento, el desinterés y la deserción como último recurso de parte del alumnado.

Propuestas de Estrategias Metodológicas que podrían disminuir los efectos de LA TRANSICIÓN

Elaborar y aplicar un plan de transición desde la Educación Intercultural Bilingüe, a la Monolingüe, que se aplicará durante el primer trimestre del año escolar, tomando en 
consideración los objetivos educacionales del grado inmediato, inferir y compararlos con los del $5^{\circ}$ grado, con el propósito de identificar los niveles de complejidad con que serán tratado los contenidos y así realizar adecuaciones a los contenidos acorde a la realidad del entorno sociocultural del educando, utilizando para su desarrollo horarios flexibles y aulas interactivas, en las que los aprendizajes lleven todo el rigor científico que requiere cada una de las asignaturas.

Se deberá hacer énfasis en el desarrollo de las habilidades lingüísticas a través de la integración de contenidos en las diversas asignaturas, ya que es de esta manera que el niño y la niña percibe los fenómenos que ocurren en la sociedad y el ambiente natural. La/el maestro(a) en el aula flexible debe ser un facilitador y asumir un liderazgo más vital, deberá movilizar situaciones de comunicación, organizando experiencias de aprendizaje, guiando la evaluación. Se deberán elegir las técnicas adecuadas con las que trabajará cada contenido para hacer realidad las potencialidades del grupo

Durante la etapa de transición se deberán propiciar aprendizajes significativos, abandonando la idea de aprendizaje memorístico, concebido como la repetición sin reflexión. El docente deberá recurrir a la reflexión para emprender a hacer que sus alumnas/os trabajen con objetos concretos, producto de su creatividad, además llevar las problemáticas a lo concreto, dramatizar situaciones con el propósito de ponerlos en contacto con los objetos de estudio.

Se sugiere proponer actividades diversas mediante estrategias y técnicas participativas, disponiendo de los materiales básicos que permitan la interiorización de los conocimientos de una manera diferente, facilitando el procedimiento de apropiación y de transferencia, a través de la búsqueda de técnicas que se adapten a cada circunstancia. Además, disponer de espacios flexibles en el aula (rincón de lectura, mapoteca, trabajo grupal, entre otros) que permitan a los estudiantes elegir y/o construir el lugar adecuado para el desarrollo del proceso educativo.

Entre algunas técnicas ${ }^{15}$ que se recomiendan utilizar durante la etapa de transición están:

\section{a. Código común}

\section{¿Qué es?}

Es una técnica de producción para las primeras clases.

\section{¿Para qué sirve?}

Para que cada grupo elabore su propio reglamento, preciando los derechos, obligaciones y las faltas que regularán la convivencia, así como también para auto controlar su cumplimiento, aplicando las sanciones que en él se establezcan.

15 En el trabajo completo se detallan éstas y otras estrategias. 


\section{b. Producción cooperativa}

\section{¿Qué es?}

Es una técnica de producción compartida.

\section{¿Para qué sirve?}

Para desarrollar, integrar y/o profundizar un tema. Para compartir la construcción del conocimiento con las compañeras/os de clase.

c. Redes

¿Qué es?

Es una técnica que permite la construcción colectiva de un saber.

\section{¿Para qué sirve?}

Para aprender o repasar cualquier tema.

¿Cómo se hace?

Previamente, el docente habrá elegido el tema que se va a trabajar, y a partir de él, habrá seleccionado uno o más textos periodísticos referidos al mismo.

\section{d. Descubriendo problemas}

\section{¿Qué es?}

Es una técnica para detectar problemas sociales del entorno y la comunidad.

\section{¿Para qué sirve?}

Para identificar y compartir una problemática social de la realidad circundante. Esta técnica se utiliza, preferentemente, para iniciar una actividad.

\section{LISTA DE REFERENCIAS}

Acunzo, Mario (1991). Educación e Identidad Étnica. Ediciones ABYA-YALA, Quito, Ecuador.. 
Carreño Huerta, Fernando (1998). Enfoques y principios teóricos de La Evaluación. Trillas, México.

Cunningham K., Mirna (1999). Evolución, Tendencias, y Principales Características de La Educación. URACCAN,.

Diccionario, Océano de La Lengua Española, Océano Editorial. 1996.

Amodio, Emanuele (1988). Educación, Escuela y culturas Indígenas de América Latina, Tomo II., ABYA YALA.

Espinoza Flores, Miriam (1994). Formulación e interpretación de Objetivos, Managua, INATEC-OIT, Netherland,.

Enciclopedia, Manual de La Educación. Océano editorial. 1998.

Freeland. Jean, Universidad Portsmouth (1994). Investigación sobre las necesidades lingüisticas del estudiante de La Costa Caribe Nicaragüense. IPILC. URACCAN.

Mialaret, Gastón, Diccionario. Ciencias de La Educación, Océano Editorial, 1999.

Montes, Emilia. Pueblos Indigenas y Educación. Abya-Yala. Perú.

Miriam Espinoza Flores, Carlos Cantú Laguna y María Solórzano, coat (1993). Aplique la Técnica de Corrillos en sus tareas Docentes. Managua, INATEC-OIT, Holanda.

Rizo, Mario (2003). Cultura, $1^{\circ}$ ed. Managua: Terra Nuova, v 1.

Sola Mendoza, Juan. Pedagogía en pildora. México, trillas 1989.

Traña Lezama, Imelda (1998). La Técnica Demostrativa en sus Estrategias Didácticas. Managua, INATEC-OIT, Holanda.

Ujukam, German, (1997). Pueblos Indigena y Educción. ABYA-YALA, Quito.

Venezia Mauceri, Paolo, (2003). Educación Intercultural y Plurilingüe. IBIS - KEPA TERRA NUOVA. 